\title{
LOS ELEMENTOS COMPOSITIVOS HIPER-/HIPO- EN EL DICCIONARIO*
}

\author{
FERNANDO PÉREZ LAGOS \\ (Universidad de Málaga)
}

\begin{abstract}
This work analyses the productivity of the hiper-and hipo- prefixes included in the Real Academia Española dictionary and in VOX'S general dictionary throughout their last five editions (halfs century of lexicographical creation). Appendix I offers each one of the words formed with these prefixes, followed by the editions in which each one appears. The organization of this material in appendix II may be used to find which words have appeared in each new edition and to compare both editions and dictionarics.
\end{abstract}

Pensamos que la presentación de la productividad de los elementos hiper- e hipoen dos importantes diccionarios generales de la lengua española, como el académico ${ }^{1}$ y el $\operatorname{VOX}^{2}$, puede resultar enriquecedora, además de propiciar futuros estudios pormenorizados.

Quizás un corpus lexicográfico no sea el que mejor refleje el estado de un fenómeno de lengua, pero sí se ha convertido en el mejor al que un investigador puede acceder con facilidad para comprobar cómo se hace eco, con todas sus limitaciones, del vocabulario empleado en un momento dado ${ }^{3}$.

\footnotetext{
" Este trabajo ha sido posible, en parte, a la vista de los materiales recogidos gracias a una ayuda del Ministerio de Educación y Ciencia (Proyecto CICYT, PB88-0323).

${ }^{1}$ Real Academia Española, Diccionario de la lengua española, $21^{\mathrm{a}}$ ed., Madrid, Espasa Calpe, 1992.

2 Diccionario General de la Lengua Española, VOX, Barcelona, Biblograf, 1997.

${ }^{3}$ Jean y Claude Dubois exponen cómo los diccionarios son testimonio de la actitud de una comunidad linguística y reflejo de un estado de lengua y, pese a sus limitaciones, se han convertido en «source d'études linguistiques portant sur l'évolution de la langue ou sur les modifications de l'attitude de la communauté à l'égard de sa propre langue». Dubois, Jean/Dubois, Claude, Introduction à la lexicographie. Le dictionnaire, París, 1971, p. 110.
} 
De todos es conocido el importante papel desempeñado por el diccionario académico como reflejo de nuestra norma lingüística, pero también es de sobra conocido que esta misma dedicación le obliga a ser excesivamente cauto y reticente, sobre todo a la hora de reflejar novedades léxicas.

Los otros diccionarios, llamados generales o de uso, existentes en el mercado, como el Diccionario General VOX, aunque por un lado siguen fielmente las consideraciones académicas, por otro tratan de paliar las «ausencias» académicas, haciéndose descriptivos del léxico general empleado en el español actual.

El limitarnos a la edición más actual de cada uno de estos diccionarios, si bien ofrecía la posibilidad de reflejar la consideración lexicográfica de hoy respecto a los dos elementos formadores de nuestro léxico, no mostraba cómo se había llegado hasta la situación del momento, y ni siquiera podíamos separar lo que pertenecía a una consideración novedosa de los últimos años de lo que estaba reflejado desde siempre.

La aparición de la primera edición del diccionario VOX en el año 1945 nos ha parecido una fecha razonable como punto de partida. Medio siglo de creación lexicográfica a través de cinco ediciones parecen suficientes para reflejar los grados de productividad que en el tiempo han tenido estos dos elementos creadores. Ya sólo cabe tomar también las cinco ediciones correspondientes del diccionario académico ${ }^{4}$.

En definitiva, hemos planteado una postura que atiende tanto a las actitudes prescriptivas (Academia) como a las descriptivas (Vox), además de posibilitar el estudio en sincronía o en diacronía. No pretendemos, por tanto, teorizar sobre un aspecto de la formación de palabras (ya en otra ocasión mostramos los problemas para la determinación de la naturaleza de este tipo de formaciones ${ }^{5}$ ), sino sólo mostrar la presencia en los diccionarios de dos clásicos elementos compositivos y comprobar su efectividad a lo largo de los últimos cincuenta años.

El conjunto de voces obtenidas, así como las distintas ediciones en las que aparecen, es ofrecido alfabéticamente en el apéndice I. A partir de los datos allí contenidos extraemos los siguientes datos:

- Los dos diccionarios ofrecen mayor número de formaciones con el elemento hipo-, que con el elemento hiper-.

\footnotetext{
${ }^{4}$ Las obras consultadas para la extracción del material tomado como punto de partida y las etiquetas utilizadas son las siguientes: V-97: Diccionario general de la lengua española, VOX, Barcelona, 1997; V-87: Diccionario general ilustrado de la lengua española, VOX, Barcelona, 1987; V-73: para la edición de esta misma obra de 1973; V-53: para la de 1953; V-45: para la primera edición de este diccionario en 1945. Para el Diccionario de la lengua española de la Real Academia Española utilizamos las claves siguientes: D-92: para la edición vigente de 1992; D-84: para la edición de 1984; D-70, para la edición de 1970; D-56: para la edición de 1956; D-47: para la edición de 1947.

${ }^{5}$ Pérez Lagos, F., «¿Entre composición y afijación? Naturaleza de los elementos de formación culta», en Estudios de Lingiuistica General (11I), Granada, 1997, págs. 361-369.
} 
- Como era de esperar, el diccionario VOX recoge gran cantidad de voces no presentadas por el diccionario académico. Concretamente 60 formaciones con hiper-, y 61 con hipo-.

- El diccionario académico ofrece en la edición actual 14 formas con hiper- y 15 creaciones con hipo- no recogidas en su edición de 1947.

- El diccionario VOX ofrece en su edición actual 75 formaciones con hiper- y 80 con hipo- que no contemplaba en su primera edición de 1945.

- Esta notable diferencia que presentan en la actualidad los dos diccionarios en cuanto al número de creaciones, no se produce desde las primeras ediciones consideradas, sino a partir de los años setenta, concretamente a partir de la tercera edición VOX (1973).

- De hecho, en las primeras ediciones, el diccionario VOX contiene menos formaciones con estos elementos que el diccionario académico.

- Por otro lado, la incorporación académica de las nuevas voces ha sido mayoritaria en las ediciones de 1956 y 1970, mientras que en las dos últimas ediciones apenas aparecen nuevas formaciones con hiper-o hipo-.

Los siguientes cuadros ofrecen el número de voces formadas con estos elementos que presenta cada edición, y con ello damos a conocer la evolución de la capacidad productiva a través de los años de cada elemento:

HIPER-

\begin{tabular}{|c|c|c|c|}
\hline \multicolumn{2}{|c|}{ DRAE } & \multicolumn{2}{c|}{ VOX } \\
\hline D-47 & 25 & 24 & V-45 \\
\hline D-56 & 30 & 26 & V-53 \\
\hline D-70 & 36 & 43 & V-73 \\
\hline D-84 & 38 & 85 & V-87 \\
\hline D-92 & 39 & 99 & V-97 \\
\hline
\end{tabular}

HIPO-

\begin{tabular}{|c|c|c|c|}
\hline \multicolumn{2}{|c|}{ DRAE } & \multicolumn{2}{c|}{ VOX } \\
\hline D-47 & 31 & 27 & V-45 \\
\hline D-56 & 38 & 28 & V-53 \\
\hline D-70 & 44 & 56 & V-73 \\
\hline D-84 & 44 & 84 & V-87 \\
\hline D-92 & 46 & 107 & V-97 \\
\hline
\end{tabular}

En el Apéndice II mostramos una de las posibles organizaciones del material ofrecido en el apéndice anterior que, por sí sola, facilita una descripción de los hechos que pretendemos reflejar con el presente trabajo. En él podemos conocer ahora cúales son las voces que han ido apareciendo en cada una de las ediciones de los dos diccionarios. Aparecen en columnas enfrentadas las formaciones con hiper-y las creaciones con hipo- y cada edición del diccionario académico aparece seguida de la edición de fecha más próxima del diccionario VOX.

Esta estructuración permite análisis muy variados: desde el tratamiento de una sola formación hasta un grupo de ellas o bien su totalidad; desde la consideración exclusivamente normativa-académica hasta la consideración de lo no considerado aún por la Academia; cada uno de los casos anteriores podría ser tratado en diacronía desde 
los años cuarenta o bien en estudios sincrónicos de la época actual o de la década elegida.

Si atendemos a los listados ofrecidos en este apéndice II, podremos comprobar, por ejemplo, cómo se reparten las voces ofrecidas en cada edición con respecto a las distintas ediciones del otro diccionario.

I) $D-47-V-45$

a) El diccionario académico recoge 25 palabras formadas con hiper- y 31 con hipoen su decimoséptima edición del año 1947:

- De las 25 creaciones académicas con el elemento hiper-, 20 de ellas ya estaban presentes en la primera edición de 1945 del diccionario VOX, hipertensión no se recoge hasta la siguiente edición del VOX, 3 más no aparecen hasta la edición de 1973 (hiperclohídrico, hiperestesiar, hipermetamorfosis) y, finalmente, hipercrítica no se recoge $e^{6}$.

- De las 31 formas con el elemento hipo-, 23 de ellas ya estaban presentes en la edición de 1945 del diccionario VOX, hiposulfürico no se recoge hasta la siguiente edición de 1953, otras 5 aparecen en la edición de 1973 (hipoclorhidria, hipoclorhídrico, hiposulfato, hiposulfito, hiposulfuroso) y 2 no son recogidas por el diccionario VOX (hipocastáneo, hipótesi).

b) El diccionario VOX, en su primera edición de 1945, recoge 24 creaciones con el formante hiper- y 27 con el elemento hipo-:

- De las 24 formas con el elemento hiper-, 20 de ellas aparecen en la edición de 1947 del diccionario académico, hipermétrope se recoge en la edición de 1956 y las 3 restantes no aparecen en el diccionario de la Academia (hiperamnesia, hipersecreción, hipertermal).

- De las 27 voces con el elemento hipo-, 23 de ellas están recogidas en la edición académica de 1947, hipocastanáceo no se recoge hasta la edición de 1956 y el resto (hipocotíleo, hipóstilo, hipotaxis) no ha aparecido aún en el diccionario académico.

II) $D-56-V-53$

a) Unos diez años más tarde, la siguiente edición académica (la décimo octava de 1956) añade 5 nuevas voces formadas con el elemento hiper- y 7 nuevas incorporaciones con el elemento hipo-:

- De la 5 nuevas voces con el elemento hiper-, una de ellas, hipermétrope, ya había aparecido en la primera edición de 1945 del diccionario VOX; las cuatro restantes (hiperestésico, hiperhidrosis, hipertermia, hipertónico) no son recogidas por el diccionario VOX hasta su tercera edición de 1973.

\footnotetext{
${ }^{6}$ Es la única entrada académica aparentemente no recogida por el diccionario VOX y se debe sólo a que este diccionario considera la forma femenina como una acepción más dentro de la entrada adjetiva.
} 
- De las 7 nuevas voces formadas con el elemento hipo-, una de ellas, hipocastanáceo, ya estaba presente en la edición de 1945 del diccionario VOX, 5 más (hipoglucemia, hipotálamo, hipotensión, hipotermia, hipotónico) no aparecen en el diccionario VOX hasta su edición de 1973, y la restante (hipotonía) será recogida en la edición VOX de 1987.

b) La edición más o menos próxima del diccionario VOX, la segunda edición, del año 1953, sólo añade dos formaciones con hiper-y una con hipo- a las ofrecidas en su primera edición:

- De las 2 nuevas formaciones con hiper-, hipertensión ya estaba presente en la edición académica de 1947; en cambio, hipertono, aún no ha sido recogida por el diccionario académico.

- El nuevo vocablo con hipo- añadido, hiposulfúrico, se encontraba ya en la edición académica de 1947.

III) $D-70-V-73$

a) El diccionario académico añadió en su edición de 19706 nuevas creaciones con hiper-y otras tantas con hipo-:

- De las 6 nuevas voces formadas con el elemento hiper-, dos de ellas, hiperoxia e hipertenso, serán incluidas en la edición de 1973 del diccionario VOX; las cuatro restantes (hiperémesis, hiperfunción, hiperplasia, hipertonía) no son contempladas hasta la edición VOX de 1987.

- De las nuevas voces formadas con hipo-, cuatro de ellas (hipocentro, hipofosfito, hipofosforoso, hipoxia) se recogen en el diccionario VOX de 1973, las dos restantes (hipofunción, hipomanía) no aparecen hasta la edición de 1987.

b) La tercera edición del diccionario VOX, de 1973, incorpora en esta ocasión un gran número de formaciones (mayor que el total incorporado por el diccionario académico en todas sus ediciones): 17 nuevas voces con hiper- y 28 con hipo-:

- De las 17 nuevas voces formadas con el elemento hiper-, 3 de ellas (hiperclohídrico, hiperestesiar, hipermetamorfosis) ya estaban en 1947 en el diccionario académico, 4 más (hiperestésico, hiperhidrosis, hipertermia, hipertónico) se incluyeron en el diccionario académico de 1956, hiperoxia e hipertenso aparecieron en la siguiente edición de 1970, mientras que hipersensibilidad e hipersensible no lo hicieron hasta la edición de 1984. Las 6 voces restantes (hiperacidez, hiperboreal, hipercorrección, hiperfocal, hipergénesis, hipergol) no se encuentran recogidas en ninguna de las ediciones académicas.

- De las 28 nuevas voces creadas con hipo-, 5 de ellas (hipoclorhidria, hipoclorhídrico, hiposulfato, hiposulfito, hiposulfuroso) ya estaban el diccionario académico de 1947, otras 5 más (hipoglucemia, hipotálamo, hipotensión, hipotermia, hipotónico) en la edición de 1956, 4 más (hipocentro, hipofosfito, hiposforoso, hipoxia) en la de 1970, 
mientras que hipotenso no ha sido contemplada hasta la edición académica actual de 1992. Las 14 voces restantes no se encuentran en ninguna edición académica.

\section{IV) $D-84-V-87$}

a) Catorce años más tarde, en su edición de 1984, el diccionario académico sólo presenta 2 nuevas voces formadas con hiper- y no añade ningún vocablo más a los ya creados con el formante hipo-:

- Los dos nuevos vocablos formados con hiper-, hipersensibilidad e hipersensible, ya aparecían en la edición de 1973 del diccionario VOX.

- Es inexplicable que el diccionario académico, después de catorce años desde su anterior edición, no realice ninguna modificación ni añadido respecto a las creaciones con el elemento hipo-que entonces presentara.

b) La edición correspondiente de los diccionarios VOX, publicada en 1987 , profundamente renovada, sí ofrece gran número de nuevas formaciones. En concreto 42 con el elemento hiper- y 28 con hipo-:

- De las 42 voces con el elemento hiper-, sólo 4 estaban ya en la edición académica de 1970 (hiperémesis, hiperfunción, hiperplasia, hipertonía) y una ha sido incluida en la última edición de 1992 (hipertiroidismo). Son, por tanto, 37 las voces presentadas por VOX en 1987 que aún no se han contemplado en las ediciones de la Academia.

- De las 28 nuevas voces formadas con hipo-, dos de ellas (hipofunción, hipomanía) fueron recogidas por el diccionario académico en 1970 y una, hipotonía, aún antes, en la edición de 1956. Las 25 voces restantes no están presentes en el diccionario académico.

V) $D-92-V-97$

a) La actual edición académica de 1992 aporta a la edición anterior una formación más con el elemento hiper- y dos con el elemento hipo-:

- El nuevo vocablo con hiper-, hipertiroidismo, ya había sido recogido en la edición de 1987 del diccionario VOX.

- De las dos creaciones con hipo-, el vocablo hipotenso también se ofrecía ya en la edición VOX de 1973, mientras que hipomaníaco no se recoge aún en los diccionarios VOX.

b) La edición del Diccionario General de VOX de 1997 incluye 14 nuevas formaciones con hiper- y 23 con el elemento hipo-:

- Ni los 14 vocablos formados con hiper-, ni los 23 formados con hipo-, recogidos por primera vez en esta edición VOX, están presentes en las ediciones académicas.

Los datos comentados sobre las distintas aportaciones de cada edición y su relación con las ediciones del otro diccionario aparece reflejada en los siguientes cuadros: 
HIPER-

\begin{tabular}{|c|c|c|c|c|c|c|}
\hline & $\begin{array}{c}\mathrm{D}-47 \\
25\end{array}$ & $\begin{array}{c}\mathrm{D}-56 \\
5\end{array}$ & $\begin{array}{c}\mathrm{D}-70 \\
6\end{array}$ & $\begin{array}{c}\mathrm{D}-84 \\
2\end{array}$ & $\begin{array}{c}\mathrm{D}-92 \\
1\end{array}$ & $\begin{array}{c}\text { NO DRAE } \\
61\end{array}$ \\
\hline $\begin{array}{c}\mathrm{V}-45 \\
24\end{array}$ & 20 & 1 & 0 & 0 & 0 & 3 \\
\hline $\begin{array}{c}\mathrm{V}-53 \\
2\end{array}$ & 1 & 0 & 0 & 0 & 0 & 1 \\
\hline $\begin{array}{c}\mathrm{V}-73 \\
17\end{array}$ & 3 & 4 & 2 & 2 & 0 & 6 \\
\hline $\begin{array}{c}\mathrm{V}-87 \\
42\end{array}$ & 0 & 0 & 4 & 0 & 1 & 37 \\
\hline $\begin{array}{c}\mathrm{V}-97 \\
14\end{array}$ & 0 & 0 & 0 & 0 & 0 & 14 \\
\hline $\begin{array}{c}\text { NO VOX } \\
1\end{array}$ & 1 & 0 & 0 & 0 & 0 & \\
\hline
\end{tabular}

$\mathrm{HIPO}-$

\begin{tabular}{|c|c|c|c|c|c|c|}
\hline & $\begin{array}{c}\mathrm{D}-47 \\
31\end{array}$ & $\begin{array}{c}\mathrm{D}-56 \\
7\end{array}$ & $\begin{array}{c}\mathrm{D}-70 \\
6\end{array}$ & $\begin{array}{c}\mathrm{D}-84 \\
0\end{array}$ & $\begin{array}{c}\mathrm{D}-92 \\
2\end{array}$ & $\begin{array}{c}\text { NO DRAE } \\
64\end{array}$ \\
\hline $\begin{array}{c}\mathrm{V}-45 \\
27\end{array}$ & 23 & 1 & 0 & 0 & 0 & 3 \\
\hline $\begin{array}{c}\mathrm{V}-53 \\
1\end{array}$ & 1 & 0 & 0 & 0 & 0 & 0 \\
\hline $\begin{array}{c}\mathrm{V}-73 \\
28\end{array}$ & 5 & 5 & 4 & 0 & 1 & 13 \\
\hline $\begin{array}{c}\mathrm{V}-87 \\
28\end{array}$ & 0 & 1 & 2 & 0 & 0 & 25 \\
\hline $\begin{array}{c}\mathrm{V}-97 \\
23\end{array}$ & 0 & 0 & 0 & 0 & 0 & 23 \\
\hline $\begin{array}{c}\text { NO VOX } \\
3\end{array}$ & 2 & 0 & 0 & 0 & 1 & \\
\hline
\end{tabular}

Aunque, en esta ocasión nos hayamos limitado a la presentación y organización de las informaciones, así como a una descripción de los datos que de ellas se desprenden, el conocimiento adquirido sobre la productividad de estos dos elementos y sobre su movimiento a través de los años, así como el poder disponer del vocabulario creado con ellos (bien en su conjunto o bien distribuido en diferentes cortes sincrónicos, o atendiendo al tipo de diccionario), evidencia la posibilidad de múltiples y variados 
estudios futuros ${ }^{7}$. Todo ello nos sirve para demostrar que como estadio previo a la elaboración de cualquier teoría es precisa la realización de una buena descripción, de una «teoría descriptiva» ${ }^{8}$.

Además de realizar la descripción de las voces que incluyen ambos diccionarios en su distintas ediciones y formadas con estos elementos, es preciso atender a los contenidos a los que hacen referencia $\mathrm{y}$, en concreto, a las principales aportaciones semántico-funcionales que realizan estos dos elementos dentro de dichas formaciones. Apuntaremos, por ahora, algunos de los aspectos principales que se desprenden de una primera observación de los contenidos de estos vocablos:

1.- En un gran número de voces en las que aparecen son calcos de formaciones griegas y, por tanto, están presentes en los dos diccionarios desde las primeras ediciones consideradas. En estas formaciones ya creadas en griego suele ofrecer el sentido original de 'por encima' / 'por debajo' como circunstancial del proceso indicado por el componente que sigue (hipérbole, hipófisis).

2.- En otras construcciones indica 'que está sobre, o más allá / que está bajo' lo que designa el segundo formante (hiperespacio, hipotálamo, hipogeo).

3.- En formaciones más modernas, sobre todo, indica 'superioridad / inferioridad en la intensidad, la cualidad o la cantidad (hipercorrección, hiperinflación, hipomanía, hiposomnia).

4.- Son abundantes los términos científicos y técnicos, especialmente médicos, en los que el sentido anterior termina formando parejas de 'exceso / escasez', 'aumento/disminución' con respecto a lo normal y ordinario.

Así, por ejemplo, se designan ciertas anormalidades patológicas, como el aumento excesivo de secreciones o sustancias orgánicas, el excesivo funcionamiento o desarrollo de una facultad, especificadas en el elemento formante o raíz griega (hiperemia, hipertonía, hiperestesia) o en el sustantivo independiente (hiperacidez, hipernutrición, hiperinmunidad, hipersecreción) a los que se unen.

En el lado contrario, especialmentente en patología y biología, encontramos con hipo- el valor de 'insuficiencia o disminución anormal' de lo especificado por el sustantivo al que acompaña (hipotensión, hipoacidez, hipocalcemia, hipocinesia, hipoclorhidria). El carácter atenuativo que adquiere el formante le permite estar siempre en disposición para la construcción de los antónimos a las formaciones con hiper-.

\footnotetext{
${ }^{7}$ Algunas de nuestras publicaciones sobre este campo de formación de palabras así lo demuestran: Formación de palabras: la composición culta en los diccionarios (DRAE-VOX), Málaga, 1997; «Los nuevos elementos compositivos en el diccionario de la Academia», en III Jornadas sobre el estudio y enseñanza del léxico (abril 1996), Granada, 1997.

${ }^{8}$ Peytard, Jean, Recherches sur la préfixation en français contemporain, París, 1975, escribe que: «ll existe une nécessaire «theorie descriptive», qui n'est qu'une étape, qu'un moment, certes, mais aussi nécessaire à la théorie générale que le sol aux pas du marcheur», pág. 66
} 
5.- Desde el sentido de anormalidad por exceso e intensidad surge la utilización de hiper- en el lenguaje común como un simple intensivo ('muy') unido sobre todo a adjetivos. Es una posibilidad siempre abierta a la creación espontánea y no presente en la nomenclatura de los dos diccionarios estudiados.

6.- En los compuestos químicos, el elemento hipo- indica 'de menos oxígeno' que el ácido o sal cuyo nombre acompaña (hipofosforoso, hiposulfato, hiposulfuroso).

\section{APÉNDICE I ÍNDICE ALFABÉTICO DE VOCES ${ }^{9}$}

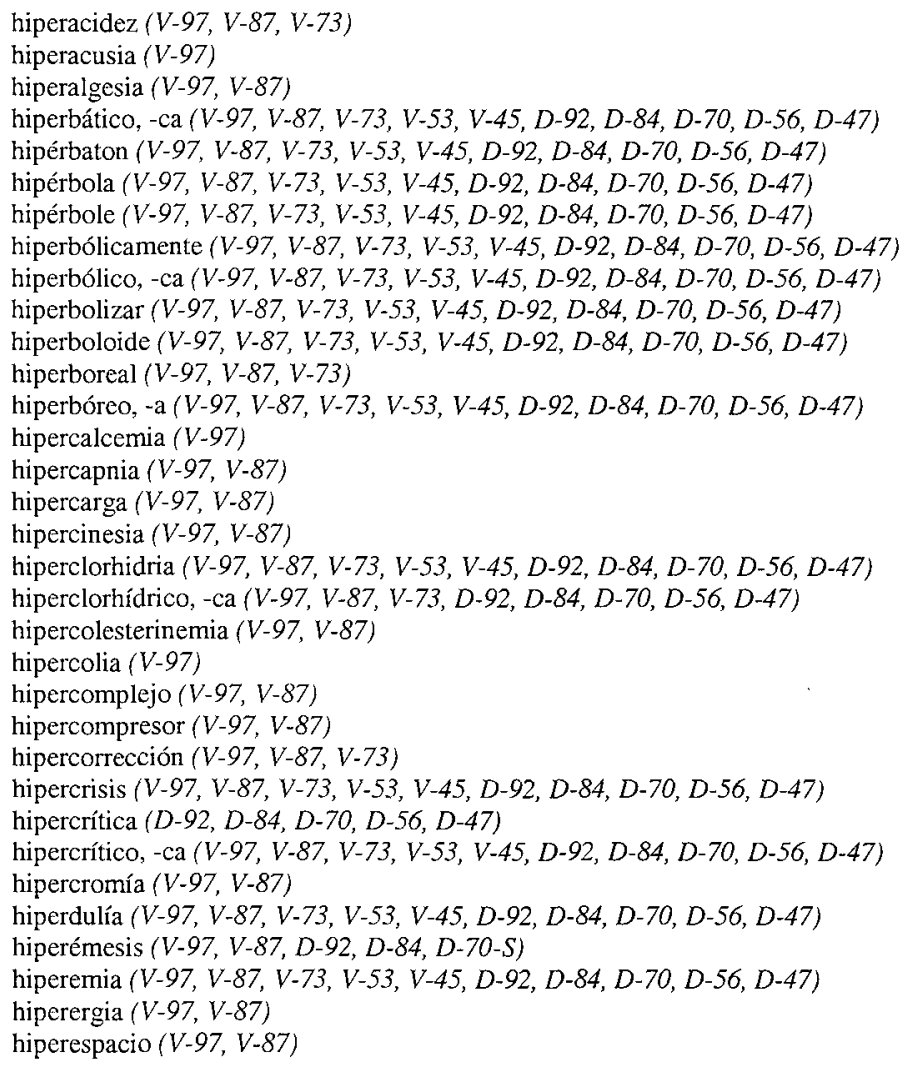

${ }^{9}$ Además de las etiquetas ya presentadas en la nota 4, distinguimos: D-47-S y D-70-S para las voces presentes en el Suplemento de la edición académica de 1947 y en el de 1970, respectivamente. 
hiperestático, -ca $(V-97, V-87)$

hiperestenia $(V-97)$

hiperestesia (V-97, V-87, V-73,V-53,V-45, D-92, D-84, D-70, D-56,D-47)

hiperestesiar ( $V-97, V-87, V-73, D-92, D-84, D-70, D-56, D-47)$

hiperestésico, -ca $(V-97, V-87, V-73, D-92, D-84, D-70, D-56)$

hiperextensión ( $V$-97)

hiperfocal $(V-97, V-87, V-73)$

hiperfunción $(V-97, V-87, D-92, D-84, D-70-S)$

hipergénesis $(V-97, V-87, V-73)$

hiperglobulia ( $V-97)$

hiperglucemia $(V-97, V-87)$

hipergol $(V-97, V-87, V-73)$

hiperhidrosis ( $V-97, V-87, V-73, D-92, D-84, D-70, D-56)$

hiperinflación ( $V-97, V-87)$

hiperinmunidad ( $V-97)$

hiperlipidemia $(V-97)$

hiperlipoproteinemia $(V-97)$

hipermenorrea $(V-97, V-87)$

hipermercado $(V-97, V-87)$

hipermetamorfosis ( $V-97, V-87, V-73, D-92, D-84, D-70, D-56, D-47)$

hipermetría ( $V-97, V-87, V-73, V-53, V-45, D-92, D-84, D-70, D-56, D-47$ )

hipermétrope ( $V-97, V-87, V-73, V-53, V-45, D-92, D-84, D-70, D-56)$

hipermetropia $(V-97, V-87, V-73, V-53, V-45, D-92, D-84, D-70, D-56, D-47)$

hipermnesia $(V-97, V-87, V-73, V-53, V-45)$

hipernefroma $(V-97, V-87)$

hipernúcleo $(V-97, V-87)$

hipernutrición $(V-97, V-87)$

hiperónimo (V-97)

hiperosmia $(V-97)$

hiperostosis $(V-97, V-87)$

hiperoxia ( $V-97, V-87, V-73, D-92, D-84, D-70-S)$

hiperparásito $(V-97, V-87)$

hiperparatiroidismo $(V-97)$

hiperpepsia $(V-97, V-87)$

hiperpituitarismo ( $V-97, V-87)$

hiperplasia $(V-97, V-87, D-92, D-84, D-70)$

hiperrealismo $(V-97, V-87)$

hipersalino, -na $(V-97)$

hipersecreción ( $V-97, V-87, V-73, V-53, V-45)$

hipersensibilidad $(V-97, V-87, V-73, D-92, D-84)$

hipersensibilización (V-97, V-87)

hipersensible (V-97,V-87,V-73, D-92, D-84)

hipersialosis $(V-97, V-87)$

hipersomía (V-97)

hipersomnia $(V-97, V-87)$

hipersónico, -ca $(V-97, V-87)$

hipersonido $(V-97, V-87)$

hiperstena $(V-97, V-87)$

hipersustentación ( $V-97, V-87)$

hipersustentador, -ra $(V-97, V-87)$ 
hipertelorismo $(V-97, V-87)$

hipertensión ( $V-97, V-87, V-73, V-53, D-92, D-84, D-70, D-56, D-47-S)$

hipertenso, -sa $(V-97, V-87, V-73, D-92, D-84, D-70)$

hipertermal $(V-97, V-87, V-73, V-53, V-45)$

hipertermia $(V-97, V-87, V-73, D-92, D-84, D-70, D-56)$

hipertérmico, -ca $(V-97, V-87)$

hipertiroidismo $(V-97, V-87, D-92)$

hipertonía $(V-97, V-87, D-92, D-84, D-70)$

hipertónico, -ca $(V-97, V-87, V-73, D-92, D-84, D-70, D-56)$

hipertono $(V-97, V-87, V-73, V-53)$

hipertricosis $(V-97, V-87)$

hipertrofia ( $V-97, V-87, V-73, V-53, V-45, D-92, D-84, D-70, D-56, D-47)$

hipertrofiar $(V-97, V-87, V-73, V-53, V-45, D-92, D-84, D-70, D-56, D-47)$

hipertrófico, -ca $(V-97, V-87, V-73, V-53, V-45, D-92, D-84, D-70, D-56, D-47)$

hiperuricemia $(V-97, V-87)$

hiperventilación $(V-97, V-87)$

hipervitaminosis $(V-97, V-87)$

hipoacidez ( $V-97, V-87, V-73)$

hipoacusia $(V-97)$

hipoalgesia $(V-97)$

hipoalimentación $(V-97)$

hipobaropatía $(V-97, V-87)$

hipobranquial (V-97)

hipocalcemia $(V-97)$

hipocalórico, -ca $(V-97)$

hipocapnia $(V-97, V-87)$

hipocastanáceo, -a $(V-97, V-87, V-73, V-53, V-45, D-92, D-84, D-70, D-56)$

hipocastáneo, -a (D-92, D-84, D-70,D-56,D-47)

hipocausto $(V-97, V-87, V-73, V-53, V-45, D-92, D-84, D-70, D-56, D-47)$

hipocentro $(V-97, V-87, V-73, D-92, D-84, D-70)$

hipocicloidal $(V-97, V-87)$

hipocicloide (V-97, V-87, V-73, V-53,V-45, D-92, D-84, D-70, D-56, D-47)

hipocinesia $(V-97, V-87)$

hipocinético, -ca $(V-97)$

hipocístide $(V-97, V-87)$

hipoclorhidria $(V-97, V-87, V-73, D-92, D-84, D-70, D-56, D-47)$

hipoclorhídrico, -ca $(V-97, V-87, V-73, D-92, D-84, D-70, D-56, D-47)$

hipoclorito $(V-97, V-87, V-73)$

hipocloroso, -sa $(V-97, V-87, V-73)$

hipocolesterinemia, hipocolesterolemia $(V-97, V-87)$

hipocolia $(V-97)$

hipocondría $(V-97, V-87, V-73, V-53, V-45, D-92, D-84, D-70, D-56, D-47)$

hipocondríaco, -ca $(V-97, V-87, V-73, V-53, V-45, D-92, D-84, D-70, D-56, D-47)$

hipocóndrico, -ca $(V-97, V-87, V-73, V-53, V-45, D-92, D-84, D-70, D-56, D-47)$

hipocondrio $(V-97, V-87, V-73, V-53, V-45, D-92, D-84, D-70, D-56, D-47)$

hipocotíleo $(V-97, V-87, V-73, V-53, V-45)$

hipocraterimorfo $(V-97, V-87)$

hipocristalino, -na $(V-97, V-87)$

hipocromía $(V-97, V-87)$

hipodactilia $(V-97)$ 
hipodérmico, -ca (V-97, V-87,V-73,V-53,V-45, D-92, D-84, D-70,D-56,D-47)

hipodermis $(V-97, V-87, V-73)$

hipodermitis $(V-97, V-87)$

hipofalangia ( $V-97)$

hipofisectomía $(V-97, V-87)$

hipófisis (V-97, V-87,V-73,V-53,V-45, D-92, D-84,D-70,D-56,D-47)

hipofosfato $(V-97, V-87, V-73)$

hipofosfito $(V-97, V-87, V-73, D-92, D-84, D-70)$

hipofosfórico $(V-97, V-87, V-73)$

hipofosforoso $(V-97, V-87, V-73, D-92, D-84, D-70)$

hipofunción (V-97, V-87, D-92, D-84,D-70)

hipogástrico, -ca (V-97, V-87,V-73,V-53,V-45, D-92, D-84, D-70,D-56, D-47)

hipogastrio $(V-97, V-87, V-73, V-53, V-45, D-92, D-84, D-70, D-56, D-47)$

hipogénesis $(V-97)$

hipogénico, -ca $(V-97, V-87, V-73, V-53, V-45, D-92, D-84, D-70, D-56, D-47)$

hipogeo, -a (V-97, V-87, V-73,V-53, V-45, D-92, D-84, D-70, D-56, D-47)

hipogeusia $(V-97)$

hipógino, -na $(V-97, V-87)$

hipoglobulia $(V-97)$

hipogloso, -sa (V-97, V-87, V-73,V-53,V-45, D-92, D-84, D-70, D-56, D-47)

hipoglucemia (V-97, V-87, V-73, D-92, D-84, D-70, D-56)

hipognato, -ta $(V-97, V-87)$

hipohidrosis ( $V-97)$

hipolimnion $(V-97, V-87)$

hipolipidemia ( $V-97)$

hipolipoproteinemia $(V-97)$

hipomagma $(V-97, V-87)$

hipomanía $(V-97, V-87, D-92, D-84, D-70)$

hipomaníaco, -ca $(D-92)$

hipomenorrea $(V-97, V-87)$

hipomoclio (V-97, V-87, V-73, V-53, V-45, D-92, D-84, D-70, D-56, D-47)

hipónimo ( $V-97)$

hipoparatiroidismo $(V-97, V-87)$

hipopepsia $(V-97, V-87)$

hipopituitarismo $(V-97, V-87)$

hipoplasia $(V-97, V-87)$

hipoproteico, -ca $(V-97)$

hiposecreción ( $V-97, V-87, V-73)$

hiposmia (V-97)

hiposódico, -ca $(V-97)$

hiposomía (V-97)

hiposomnia (V-97)

hipostasis $(V-97, V-87)$

hipostenia $(V-97, V-87, V-73)$

hipostesia $(V-97, V-87)$

hipóstilo, -la (V-97, V-87, V-73,V-53,V-45)

hipostómato, -ta $(V-97, V-87, V-73)$

hiposulfato $(V-97, V-87, V-73, D-92, D-84, D-70, D-56, D-47)$

hiposulfito ( $V-97, V-87, V-73, D-92, D-84, D-70, D-56, D-47)$

hiposulfúrico $(V-97, V-87, V-73, V-53, D-92, D-84, D-70, D-56, D-47-S)$ 
hiposulfuroso (V-97, $V-87, V-73, D-92, D-84, D-70, D-56, D-47)$

hipotálamo $(V-97, V-87, V-73, D-92, D-84, D-70, D-56)$

hipotaxis $(V-97, V-87, V-73, V-53, V-45)$

hipoteca $(V-97, V-87, V-73, V-53, V-45, D-92, D-84, D-70, D-56, D-47)$

hipotecable ( $V-97, V-87, V-73, V-53, V-45, D-92, D-84, D-70, D-56, D-47)$

hipotecar (V-97, $V-87, V-73, V-53, V-45, D-92, D-84, D-70, D-56, D-47)$

hipotecario, -ria $(V-97, V-87, V-73, V-53, V-45, D-92, D-84, D-70, D-56, D-47)$

hipotensión ( $V-97, V-87, V-73, D-92, D-84, D-70, D-56$ )

hipotenso, -sa $(V-97, V-87, V-73, D-92)$

hipotensor $(V-97, V-87)$

hipotenusa ( $V-97, V-87, V-73, V-53, V-45, D-92, D-84, D-70, D-56, D-47)$

hipotermia $(V-97, V-87, V-73, D-92, D-84, D-70, D-56)$

hipotérmico, -ca $(V-97, V-87)$

hipótesis (V-97, V-87, V-73, V-53,V-45,D-92, D-84, D-70,D-56,D-47)

hipótesi ( $D-92, D-84, D-70, D-56, D-47)$

hipotéticamente (V-97, V-87, V-73,V-53,V-45, D-92, D-84, D-70,D-56, D-47)

hipotético, -ca $(V-97, V-87, V-73, V-53, V-45, D-92, D-84, D-70, D-56, D-47)$

hipotiposis $(V-97, V-87, V-73, V-53, V-45, D-92, D-84, D-70, D-56, D-47)$

hipotiroidismo $(V-97, V-87, V-73)$

hipotonía ( $V-97, V-87, D-92, D-84, D-70, D-56)$

hipotónico, - Ca $(V-97, V-87, V-73, D-92, D-84, D-70, D-56)$

hipotrofia $(V-97, V-87, V-73)$

hipoventilación $(V-97)$

hipovitaminosis $(V-97, V-87, V-73)$

hipovolemia $(V-97, V-87)$

hipoxia $(V-97, V-87, V-73, D-92, D-84, D-70-S)$

hipural $(V-97, V-87, V-73)$

\section{APÉNDICE II}

$$
\text { I, a) } D-47
$$

25

hiperbático

hipérbaton

hipérbola

hipérbole

hiperbólicamente

hiperbólico

hiperbolizar

hiperboloide

hiperbóreo

hiperclorhidria

hiperclorhídrico

hipercrisis

hipercrítica

hipercrítico

hiperdulía

hiperemia

hiperestesia

hiperestesiar
31

hipocastáneo

hipocausto

hipocicloide

hipoclorhidria

hipoclorhídrico

hipocondría

hipocondríaco

hipocóndrico

hipocondrio

hipodérmico

hipófisis

hipogástrico

hipogastrio

hipogénico

hipogeo

hipogloso

hipomoclio

hiposulfato 
hipermetamorfosis

hipermetría

hipermetropía

hipertensión

hipertrofia

hipertrofiar

hipertrófico hiposulfito

hiposulfúrico

hiposulfuroso

hipoteca

hipotecable

hipotecar

hipotecario

hipotenusa

hipótesis

hipótesi

hipotéticamente

hipotético

hipotiposis

I, b) $V-45$

24

hiperbático

hipérbaton

hipérbola

hipérbole

hiperbólicamente

hiperbólico

hiperbolizar

hiperboloide

hiperbóreo

hiperclorhidria

hipercrisis

hipercrítico

hiperdulía

hiperemia

hipermetría

hipermétrope

hipermetropía

hipermnesia

hipersecreción

hipertermal

hipertrofia

hipertrofiar

hipertrófico hiperestesia

27

hipocastanáceo

hipocausto

hipocicloide

hipocondría

hipocondríaco

hipocóndrico

hipocondrio

hipocotíleo

hipodérmico

hipófisis

hipogástrico

hipogastrio

hipogénico

hipogeo

hipogloso

hipomoclio

hipóstilo

hipotaxis

hipoteca

hipotecable

hipotecar

hipotecario

hipotenusa

hipótesis

hipotéticamente

hipotético

hipotiposis

II, a) 0.56

hiperestésico
7 hipocastanáceo 
hiperhidrosis

hipermétrope

hipertermia

hipertónico hipoglucemia

hipotálamo

hipotensión

hipotermia

hipotonía

hipotónico

II, b) $V-53$

2

hipertensión

hipertono

III, a) D-70

$$
6
$$

hiperémesis

hiperfunción

hiperoxia

hiperplasia

hipertenso

hipertonía

III, b) $V-73$

17

hiperacidez

hiperboreal

hiperclorhídrico

hipercorrección

hiperestesiar

hiperestésico

hiperfocal

hipergénesis

hipergol

hiperhidrosis

hipermetamorfosis

hiperoxia

hipersensibilidad

hipersensible

hipertenso

hipertermia

hipertónico
1

hiposulfúrico
6

hipocentro

hipofosfito

hipofosforoso

hipofunción

hipomanía

hipoxia
28

hipoacidez

hipocentro

hipoclorhidria

hipoclorhídrico

hipoclorito

hipocloroso

hipodermis

hipofosfato

hipofosfito

hipofosfórico

hipofosforoso

hipoglucemia

hiposecreción

hipostenia

hipostómato

hiposulfato

hiposulfito

hiposulfuroso

hipotálamo

hipotensión

hipotenso

hipotermia

hipotiroidismo 
hipotónico

hipotrofia

hipovitaminosis

hipoxia

hipural

IV, a) $D-84$

2

hipersensibilidad

hipersensible

IV, b) $V-87$

\section{2}

hiperalgesia

hipercapnia

hipercarga

hipercinesia

hipercolesterinemia

hipercomplejo

hipercompresor

hipercromía

hiperémesis

hiperergia

hiperespacio

hiperestático

hiperfunción

hiperglucemia

hiperinflación

hipermenorrea

hipermercado

hipernefroma

hipernúcleo

hipernutrición

hiperostosis

hiperparásito

hiperpepsia

hiperpituitarismo

hiperplasia

hiperrealismo

hipersensibilización

hipersialosis

hipersomnia

hipersónico

hipersonido

hiperstena

hipersustentación

hipersustentador

hipertelorismo
28

hipobaropatía

hipocapnia

hipocicloidal

hipocinesia

hipocístide

hipocolesterinemia

hipocraterimorfo

hipocristalino

hipocromía

hipodermitis

hipofisectomía

hipofunción

hipógino

hipognato

hiplimnion

hipomagma

hipomanía

hipomenorrea

hipoparatiroidismo

hipopepsia

hipopituitarismo

hipoplasia

hipostasis

hipostesia

hipotensor

hipotérmico

hipotonía

hipovolemia 
hipertérmico

hipertiroidismo

hipertonía

hipertricosis

hiperuricemia

hiperventilación

hipervitaminosis

V, a) D-92

1

hipertiroidismo

2

hipomaníaco

hipotenso

V, b) V-97

14

23

hiperacusia

hipercalcemia

hipercolia

hiperestenia

hiperextensión

hiperglobulia

hiperinmunidad

hiperlipidemia

hiperlipoproteinemia

hiperónimo

hiperosmia

hiperparatiroidismo

hipersalino

hipersomía

hipoacusia

hipoalgesia

hipoalimentación

hipobranquial

hipocalcemia

hipocalórico

hipocinético

hipocolia

hipodactilia

hipofalangia

hipogénesis

hipogeusia

hipoglobulia

hipohidrosis

hipolipidemia

hipolipoproteinemia

hipónimo

hipoproteico

hiposmia

hiposódico

hiposomía

hiposomnia

hipoventilación 